\title{
Articles
}

\section{Diabetes in the parents of children with Type I diabetes}

\author{
I. F. Douek, K. M. Gillespie, P. J. Bingley, E. A. M. Gale \\ Diabetes and Metabolism, Division of Medicine, University of Bristol, Bristol, UK
}

\section{Abstract}

Aims/hypothesis. Previous studies have reported an excess of Type II (non-insulin-dependent) diabetes mellitus in parents of children with Type I (insulindependent) diabetes mellitus. We set out to characterise the clinical and immunogenetic features of diabetes in parents of affected children, and to test the hypothesis that there is no excess of Type II diabetes within this population.

Methods. Clinical details were collected from 3164 parents of 1641 children with Type I diabetes participating in the Bart's-Oxford study of childhood diabetes. Islet cell antibodies, antibodies to GAD and IA-2, and HLA class II genotype were determined in a subset of this group. Individuals were assigned a classification of Type I diabetes on the basis of clinical features and measurement of islet autoantibodies. Results. Of 184 parents with diabetes, 138 (75\%) were on insulin. At least one islet autoantibody was detected in $90(59 \%)$ of 152 parents tested, and of 116 who were HLA-typed, 23 (20\%) had the highest risk genotype HLA-DRB1*03-DQA1*0501DQB1*0201 / DRB1*04-DQA1*0301-DQB1*0302. Of 46 non-insulin-treated parents, 12 had islet autoantibodies. Of all parents, 141 (4.5\%) were therefore classified as having Type I diabetes, and $31(0.98 \%)$ as Type II diabetes; 12 could not be classified because of missing data or samples.

Conclusion/interpretation. Autoimmune diabetes can present late and without immediate need for insulin treatment in parents of children with the disease. Previous studies have categorised this as Type II diabetes. Our study suggests that there is no excess of non-autoimmune diabetes in the families of children with Type I diabetes. [Diabetologia (2002) 45:495-501]

Keywords Type I diabetes, Type II diabetes, classification, familial risk, islet autoantibodies, HLA.
It has long been noted that late onset diabetes is common in the parents and siblings of individuals who develop diabetes before the age of 30 [1]. In keeping with this, later studies have shown an apparent overlap between Type I and Type II diabetes in these families [2-7]. Type II diabetes has been observed

Received: 7 November 2001 and in revised form: 3 January 2002

Corresponding author: E. A. M. Gale, Diabetes and Metabolism, Medical School Unit, Southmead Hospital, Bristol, BS10 5NB, UK, e-mail: edwin.gale@bristol.ac.uk

Abbreviations: BOX Bart's-Oxford study of childhood diabetes; JDF, Juvenile Diabetes Foundation more often in relatives of individuals with Type I diabetes than in control groups [3, 4], and siblings of children with Type I diabetes are more likely to be affected if a parent has Type II diabetes [5, 6]. An overlap between Type I and Type II diabetes was also suggested by a study of probands with Type II diabetes in which those with a family history of Type I diabetes showed a phenotype more characteristic of Type I diabetes [7]. These observations indicate a genetic link between Type I and Type II diabetes but the method of phenotyping used in previous studies requires further consideration.

Classification of diabetes by treatment $[8,9]$ has now been superseded by classification according to cause $[10,11]$. As a result, it is now recognised that 
autoimmune diabetes should no longer be considered synonymous with a need for insulin therapy, and can present at any age. Helpful though the new classification is, it is not always easy to apply to individuals. There are no specific tests for Type II diabetes, which remains largely a diagnosis of exclusion, while tests for markers of autoimmune diabetes are not widely available and can give equivocal results [12]. Most clinicians therefore still rely on the traditional indications of age, adiposity and perceived need for insulin, and these remain the basis for much of the literature.

Our aim was to carry out a systematic analysis of all parents with a diagnosis of diabetes in a large family study and to test the hypothesis that there is no excess of Type II diabetes in the parents of children with Type I diabetes.

\section{Subjects and methods}

The Bart's Oxford (BOX) study of childhood diabetes is a prospective population-based family study, which has recruited $88 \%$ of the families of children who have developed Type I diabetes under the age of 21 in the former Oxford Regional Health Authority Area since 1985 [13]. By the end of December 2000, 1641 families had been recruited into this study with regular follow up on $79 \%$ of these. At study entry, after consent was obtained, a detailed family history of diabetes was taken and blood collected for autoantibody assessment and genotyping. Mouth swabs were used for genetic analysis if an individual did not wish to give blood. The families were contacted annually to obtain information about any new cases of diabetes in first-degree relatives. The study has been approved by the Local Research Ethics Committees in the Oxford region.

At the time of analysis 3175 parents, 1612 mothers and 1563 fathers had been recruited (Fig. 1). Median age was 45.3 years (range 21.3-93.9). Eleven of these were excluded from analysis because it was not known whether they had diabetes. Of the remainder, 192 had a diagnosis of diabetes. Those presenting under the age of 15 were assumed to have classic Type I diabetes with an immediate requirement for insulin. Those diagnosed over this age were contacted for more detailed information about clinical presentation, confirmation of age at diagnosis and time to insulin treatment. Five mothers with a history of gestational diabetes that had not recurred following pregnancy were excluded, together with three other parents who were lost to follow up. The remaining 184 parents are described in this report. Classification was based on current treatment, time from diagnosis to insulin treatment, presence of islet autoantibodies, and age at diagnosis. Islet cell antibodies (ICA) and antibodies to glutamic acid decarboxylase (GAD) and the protein tyrosine phosphatase IA-2 were assessed on the first available serum sample; no sample was available on 32 parents. HLA typing for $D R B 1, D Q A 1$ and $D Q B 1$ alleles was additionally carried out on 116 parents.

Assays. ICA were measured in sera by indirect immunofluorescence [14]. End point titres were converted to Juvenile Diabetes Foundation (JDF) units by comparison with a standard curve of $\log _{2}$ JDF units versus $\log _{2}$ of the end point titre of the standard sera. The threshold of ICA detection was five JDF units. GAD and IA-2 autoantibodies were measured by radioimmunoassay [14], and considered positive if $\geq 97.5^{\text {th }}$ centile of a control population of schoolchildren. The GAD antibody as-

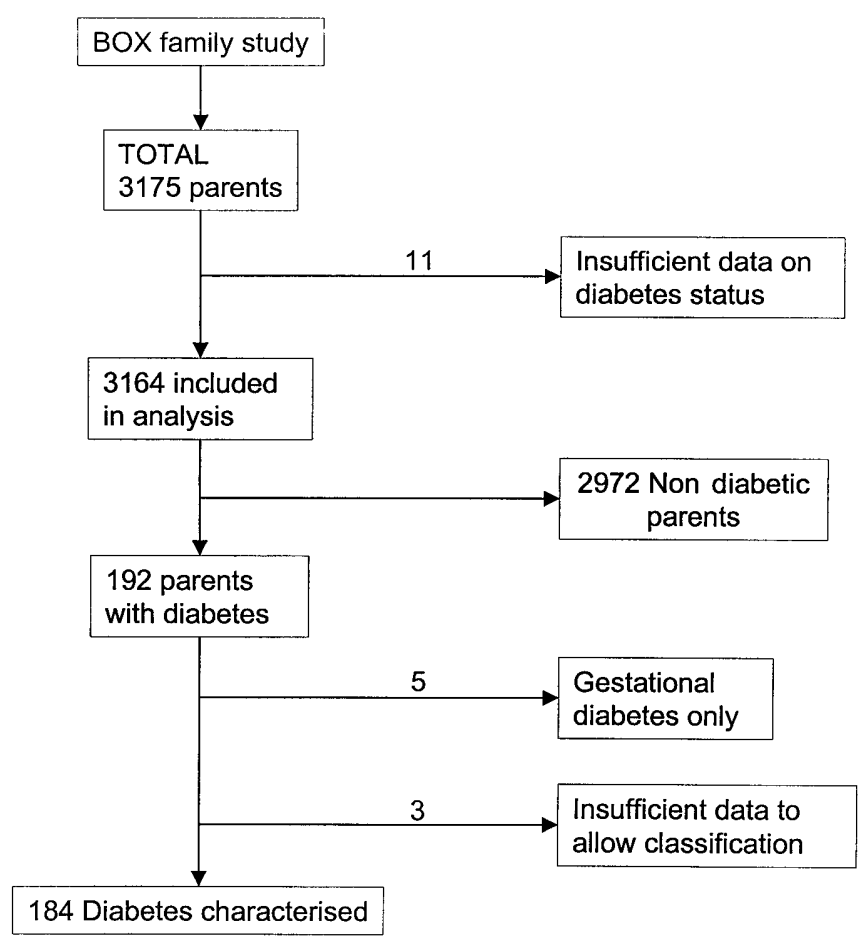

Fig. 1. Parents participating in the BOX family study

say achieved $91 \%$ sensitivity with $99 \%$ specificity, and the IA2 antibody assay reached $74 \%$ sensitivity with $99 \%$ specificity in the first Immunology of Diabetes Society (IDS) combined antibody workshop [15].

HLA genotyping was carried out on DNA from blood or mouthswab samples. Details of DNA extraction methods and HLA class II analysis have been published [16]. Briefly, mouth swab extractions were carried out using a guanidium chloride/ phenol:chloroform method. DNA was extracted from blood using a salting out method [17]. DNA quantitation was achieved using $\mathrm{OD}_{260}$ readings for blood DNA and picoGreen analysis for mouth swab DNA. Low yield DNA samples from mouth swabs underwent whole genome amplification by primer extension pre-amplification. HLA analysis was carried out by polymerase chain reaction using sequence specific primers [18]. PCR products were separated on $2 \%$ agarose containing $0.002 \%$ ethidium bromide and visualised on a dual-intensity gel documentation system. (Ultra Violet Products, Cambridge, UK). HLA typing for DRB1*01-10, for DQA $1 * 0201,0301,0302$ and 0501, and for subtypes of DQB1*02-06 was done. Extended HLA genotypes were constructed. Individuals who did not have the high risk haplotypes DRB1*03-DQA1*0501-DQB1*0201 or DRB1*04DQA1*0301-DQB1*0302 or the protective haplotype DRB1*02-DQB1*0602 were reported as DRX.

Statistical analysis. The cumulative risk of diabetes was estimated by life table analysis using the Statistics Package for Social Sciences (SPSS, Chicago, Ill., USA). The follow up period for each parent was calculated from date of birth to date of diagnosis of diabetes, or to date of last contact or death in those parents without diabetes. Risk of diabetes was then calculated from these data in yearly age bands up to age 60 . The cumulative risk figures were then analysed by assigned diabetes type and by gender.

Diabetes was classified according to the criteria shown in Figure 2. Parents who had started insulin treatment within 


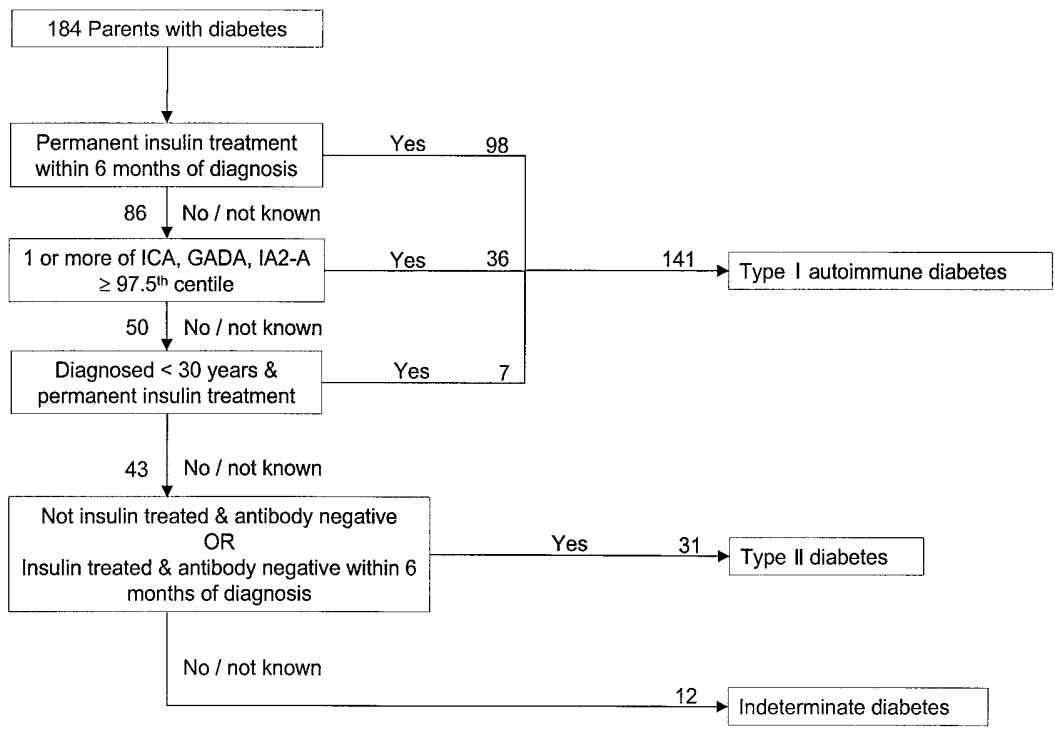

Fig. 2. Criteria used for the classification of diabetes type

6 months of diagnosis, or who had antibodies associated with autoimmune diabetes, or were permanently insulin treated and below the age of 30 at diagnosis were classified as having Type I diabetes. Parents were classified as having Type II diabetes if they were antibody negative and diet or tablet treated or if they were insulin treated and antibody negative in samples taken within 6 months of diagnosis.

\section{Results}

The analysis was performed in 184 parents with diabetes. Because both parents had diabetes in seven families, 177 of 1641 families $(10.8 \%)$ had at least one affected parent. Using life table analysis the estimated cumulative risk of parents developing diabetes by the age of 60 was $9.7 \%$ (95\%-CI 7.6-11.8); $11.5 \%$ (8.9-14.2) for fathers and $7.7 \%(3.9-11.5)$ for mothers.

The characteristics of parents with diabetes are shown in Table 1. There was an overall male-to-female ratio of $1.9: 1,75 \%$ of parents were insulin treated, $59 \%$ had one or more islet antibody at the time of sampling and $20 \%$ had the highest risk HLA genotype DRB1*03-DQA1*0501-DQB1*0201 / DRB1*04-DQA1*0301-DQB1*0302. This genotype was found in $19 \%$ of fathers and $21 \%$ of mothers. Of the 116 parents tested $4 \%$ had the protective HLA haplotype DRB1*02-DQB1*0602.

Simple classification based on treatment with insulin within 6 months of diagnosis would have given a classification of Type I diabetes in 98 parents $(3.1 \%$ of the total) with an estimated cumulative risk by age 60 of $3.4 \%$ (95\%-CI 2.7-4.1), and of Type II diabetes in 65 parents $(2.1 \%$ of the total $)$, with an esti- mated cumulative risk by age 60 of $5.8 \%(95 \%$-CI 3.8-7.8). In contrast, using the criteria shown in Figure 2,141 parents $(4.5 \%$ of the total) were classified as having Type I diabetes with an estimated cumulative risk by age 60 of $5.7 \%$ (95\%-CI 4.5-7.0), and 31 parents $(0.98 \%$ of the total) as having Type II diabetes, with an estimated cumulative risk by age 60 of $2.8 \%$ (95\%-CI 1.5-4.0). On this basis, $8.6 \%$ of probands have a parent with Type I diabetes and $1.9 \%$ have a parent with Type II diabetes. BMI was available on 21 parents with Type II diabetes, 16 of whom were overweight $(\mathrm{BMI}>25)$. Characteristics of each group are shown in Table 2.

We were not able to classify ten fathers and two mothers. These included three who were insulintreated but antibody negative when tested 2.2, 8 and 13 years after diagnosis. These were diagnosed at 38 , 41 and 50 years of age and started insulin respectively 4 years, 8 years and at an unknown period after diagnosis. HLA type was determined in two, one of whom was heterozygous for DRB $1 * 04-\mathrm{DQA} 1 * 0301$ DQB1*0302. The antibody status of the remaining nine parents was not known. Of these, five remained on diet or tablets after 2 years of diabetes, two had been converted to insulin more than 2 years after diagnosis, one of whom was homozygous for DRB1*03-DQA1*0501-DQB1*0201 and in two, time to insulin treatment was not known.

\section{Discussion}

Although the principles used for classification of Type I diabetes are widely agreed, there is no generally accepted procedure for applying them to a cohort such as ours. We therefore adopted a hierarchical approach (Fig. 2). The most robust criterion was assumed to be an early and permanent need for insulin, and individuals who started this treatment within 
Table 1. Baseline characteristics of parents with diabetes

\begin{tabular}{lc}
\hline Characteristic & $n(\%$ of those tested) \\
\hline Gender (male:female) & $120: 64$ \\
Median age at diagnosis (years) & $27.6(0.9-69)$ \\
Median duration of diabetes (years) & $16.0(0.7-55)$ \\
Current treatment & \\
Insulin: & \\
$\leq 6$ months after diagnosis & $97(53)$ \\
$>6$ months after diagnosis & $19(10)$ \\
unknown time to insulin & $22(12)$ \\
Diet / oral hypoglycaemic agents & $46(25)$ \\
Antibody status (152 tested) & \\
No antibodies & $62(41)$ \\
GAD antibodies $\geq 97.5^{\text {th }}$ centile & $71(47)$ \\
IA-2 antibodies $\geq 97.5^{\text {th }}$ centile & $50(33)$ \\
ICA antibodies $\geq 97.5^{\text {th }}$ centile & $33(22)$ \\
One or more markers & $90(59)$ \\
Two or more markers & $47(31)$ \\
HLA class II (116 tested) & \\
$D R 3 / D R 4$ & $23(20)$ \\
$D R 4 / D R 4$ & $15(13)$ \\
$D R 3 / D R 3$ & $9(8)$ \\
$D R 4 / D R X$ & $34(29)$ \\
$D R 3 / D R X$ & $12(10)$ \\
$D R B 1 * 02-D Q B 1 * 0602 / X$ & $5(4)$ \\
None of the above & $18(16)$ \\
\hline$D R 3$ &
\end{tabular}

${ }^{\mathrm{a} D R 3}$ indicates the extended haplotype DRB1*03-DQA1 *0501-DQB1*0201

$D R 4$ indicates the extended haplotype DRB1*04-DQA1 *0301-DQB1*0302

Table 2. Characteristics by assigned classification

\begin{tabular}{lll}
\hline Characteristic & $\begin{array}{l}\text { Type I diabetes } \\
(n=141)\end{array}$ & $\begin{array}{l}\text { Type II diabetes } \\
(n=31)\end{array}$ \\
\hline Male:female ratio & $1.9: 1$ & $1.4: 1$ \\
Median age at diagnosis (range) & $21.2(0.9-64)$ & $47.1(40-69)$ \\
Tested for HLA ${ }^{\mathrm{a}}(n)$ & 92 & 21 \\
$D R 3 / D R 4$ & 22 & 1 \\
$D R 4 / 4$ or $D R 4 / X$ & 39 & 6 \\
$D R 3 / 3$ or $D R 3 / X$ & 20 & 3 \\
DRB $1 * 02-\mathrm{DQB} 1 * 0602 / \mathrm{X}$ & 1 & 4 \\
None of the above & 10 & 7 \\
\hline
\end{tabular}

${ }^{\mathrm{a} D R 3}$ indicates the extended haplotype DRB1*03-DQA1 *0501-DQB1*0201

DR4 indicates the extended haplotype DRB1*04-DQA1 *0301-DQB1*0302

6 months of diagnosis were therefore assigned a classification of Type I diabetes. The remainder were then assessed according to autoantibody status, and those with one or more islet autoantibodies were added to the Type I category. Age at diagnosis was considered next, and those diagnosed under 30 years of age with a permanent need for insulin were also assigned to the Type I category. HLA Class II type was not used as a criterion for classification because HLA susceptibility alleles will inevitably be over-represented in the parents of children with diabetes.

Insulin is normally started at diagnosis in children with diabetes, but this is not necessarily the case when autoimmune diabetes presents in older age groups in association with sub-acute insulin deficiency. Autoantibodies are considered a reliable indicator of an autoimmune disease process directed against islet tissue, and ICA have been reported in up to $90 \%$ of patients with classic Type I diabetes, while antibodies to GAD are found in $85 \%$ and to IA- 2 in $75 \%$ $[14,19-21]$. Older patients are more likely to have ICA or GAD antibodies alone or in combination [20]. Antibodies tend to decrease over time [22] especially in those who develop the disease early in life [23], but GAD antibodies can persist for many years after diagnosis [24]. Adults with clinical features of Type II diabetes who have ICA or antibodies to GAD or both share the phenotype of classical Type I diabetes, in that they are non-obese with high $\mathrm{HbA}_{1 \mathrm{c}}$ concentrations and decreased beta-cell function [25], and are more likely to go on to insulin therapy [25-27]. We used the presence of one or more autoantibodies as the basis for diagnosis of autoimmune diabetes in parents who were not insulin treated or did not start insulin within 6 months of diagnosis. Conversely, absence of antibodies in such individuals was taken to indicate Type II diabetes.

In some parents, age at diagnosis was known but information as to the time to insulin treatment or antibody status was lacking. To these, we applied our third and least robust criterion, which was permanent insulin treatment following diagnosis under 30 years of age. This added a further seven individuals to the Type I category (Fig. 2). A Danish study has shown a strong association between diagnosis below 30 years of age and permanent insulin treatment, and follow up of their group revealed negligible insulin secretion in all but one of 577 individuals [28]. Diagnosis under 30 years of age with a requirement for insulin is still strong presumptive evidence of Type I diabetes in European populations, although the number of cases of Type II diabetes in this age group seems set to increase [29].

Those who could not be classified included three parents treated with insulin after 6 months who were antibody negative in samples taken more than 2 years after diagnosis. There was no way of knowing whether antibodies would have been present at diagnosis. In contrast, two other parents were antibody negative within 6 months of diagnosis and were classed as having Type II diabetes, although they subsequently required insulin.

A classification of Type I diabetes could therefore be assigned to 141 parents. A number of other characteristics reinforced this, including male predominance, which is characteristic of Type I diabetes in young adult life [30], and which would also be expected in this cohort because affected fathers are more likely than affected mothers to transmit diabetes to their offspring. The increased frequency of HLA DRB1*03-DQA1*0501-DQB1*0201／ＤRB1*04- 
DQA1*0301-DQB1*0302, the strongest susceptibility genotype for Type I diabetes [31], also supports the classification (Table 2). Although Type II diabetes was largely a diagnosis of exclusion, most of the individuals in this category were also overweight.

As noted earlier, previous studies have generally classified familial diabetes according to treatment. On the basis of early insulin therapy alone, 98 of our parents $(3.1 \%)$ would be said to have Type I diabetes and $65(2.1 \%)$ would be said to have Type II diabetes, and our report would thus be consistent with the previous literature. We have now shown that more detailed phenotyping raises the estimated prevalence of Type I diabetes to $4.5 \%$, and reduces that of Type II diabetes to $0.98 \%$. Is there then an excess of Type II diabetes in our cohort? There is no simple answer to this because estimates of the population prevalence of Type II diabetes in the UK population [32-34] are based on classification by treatment. It could, however, be noted that the cumulative risk of Type I diabetes by age 60 within our cohort is $5.7 \%$, some 10 times higher than that of the background population, whereas the cumulative risk of Type II diabetes by 60 years of age is $2.8 \%$. A study in a predominantly Caucasian population from London found a prevalence of known diabetes of $4.7 \%$ for men and $3.6 \%$ for women between 55 and 59 years of age [35] and a recent study from Poole, a town in the South of England with an ethnic mix comparable to that of the Oxford region, found values of $2.2 \%$ for men and $1.8 \%$ for women between 50 and 59 years of age and $5.4 \%$ and $3.7 \%$ respectively for the age group 60-69 years; the proportion of patients with Type I diabetes (defined as an early requirement for insulin) in these age groups is not stated, but was $18 \%$ for all ages [32]. Although precise comparison is not possible, the $2.8 \%$ cumulative risk of Type II diabetes by 60 years of age within our intensively studied family members thus falls within the range of recent estimates from comparable populations in the UK.

A systematic classification procedure supported the hypothesis that Type II diabetes was not over-represented in this large family study. Previous studies therefore need to be interpreted in the light of the classification used. One study reported 28 families with a Type II parent and one or more Type I children but the classification appears to have been based on treatment alone [2]. Genetic analysis in 11 of these families showed that in nine cases the affected parent had transmitted the HLA-DR4 allele to the child with diabetes, an observation that would be easy to explain if the affected parent had a form of autoimmune diabetes. A study based on the Swedish childhood diabetes registry reported that $8.5 \%$ of affected children had a parent with Type I diabetes and $1.7 \%$ had a parent with Type II diabetes [4]. Here again the assignment of type of diabetes was based on treatment alone, and the observation that non-insulin- treated diabetes in the parent increased the risk of insulin-treated diabetes in the offspring could be explained on the basis of misclassification. Other studies reporting that non-insulin treated diabetes in the parent is a risk factor in the child could be interpreted on the same basis [3, 5], as could the Pittsburgh Study, which reported an equal rate of insulintreated and non-insulin-treated diabetes in parents of children with diabetes [6]. In contrast, the rate of adult onset diabetes in the grandparents of children with ketosis-prone insulin-dependent diabetes has been reported to be the same as in grandparents of control children [36].

Finally, the interpretation proposed here is consistent with observations made in a population-based family study of Type II diabetes. One study reported on 1599 patients with a diagnosis of Type II diabetes [7]. Of these, 148 had no family history of diabetes, 1211 had a family history of Type II diabetes and 240 had a family history of both Type I and Type II diabetes. Those with a mixed family history differed in phenotype from the remainder. There was an increased frequency of GAD antibodies and of HLADQB1 alleles associated with Type I diabetes, a higher rate of progression to insulin treatment and less hypertension and cardiovascular disease. The difference in phenotype could not be explained simply on the basis of latent autoimmune diabetes because the same findings emerged from subanalysis of the GAD antibody-negative patients. These families therefore seem to be aetiologically distinct from the remainder, and despite the limitations of current means of classification would fit more readily within the spectrum of autoimmune diabetes.

The distinction in aetiology between the two main forms of idiopathic diabetes was one of the great achievements of diabetes research in the twentieth century [37], but the ability of current tests to distinguish the two forms of diabetes is limited. It is, however, clear that autoimmune diabetes is not just, or even predominantly, a disease of childhood and can present at any age. One study has suggested that the lifetime risk of Type I diabetes in the Danish population could be as high as $1.6 \%$ [38]. Furthermore, sub-acute presentation is common in later life, and an early requirement for insulin is not a reliable marker of the condition. Careful dissection of phenotype coupled with family studies should form the most reliable basis for laboratory studies into the causation of both forms of diabetes. Our observation suggests that there is no aetiological overlap between the conditions, and reinforces the concept of genetic heterogeneity [39].

Acknowledgements. This study was supported by Diabetes UK. P. J. Bingley was funded by the Juvenile Diabetes Foundation International. We acknowledge the continuing help and support of the children and their families; of the study fieldworkers; and of paediatricians, physicians, and diabetes nurse 
specialists in the Oxford region. We also wish to thank the project administrators, I. Wilson and S. Weeks and the BOX study fieldworkers for all their help. We are grateful to A. Williams, A. Norcross, R. Dix and R. Langley for technical assistance.

\section{References}

1. Harris H (1950) The familial distribution of diabetes mellitus: a study of the relatives of 1241 diabetic propositi. Eugenics 15: 96-119

2. Rich SS, Panter SS, Goetz FC, Helund B, Barbosa J (1991) Shared genetic susceptibility of Type I (insulin dependent) and Type II (non-insulin dependent) diabetes mellitus: contributions of HLA and haptoglobin. Diabetologia 34: 350-355

3. Quatraro A, Consoli G, Magno M, Caretta F, Ceriello A, Guigliano D (1990) Analysis of diabetic family connection in subjects with insulin-dependent diabetes mellitus. Diabetes Metab 16: 449-452

4. Dahlquist G, Blom L, Tuvemo T, Nystrom L, Sandstrom A, Wall S (1989) The Swedish childhood diabetes study - results from a nine year case register and a one year case-referent study indicating that Type I (insulin-dependent) diabetes mellitus is associated with both Type II (non-insulindependent) diabetes mellitus and autoimmune disorders. Diabetologia 32: 2-6

5. Chern MM, Anderson VE, Barbosa J (1982) Empirical risk of insulin-dependent diabetes (IDD) in sibs. Further definition of genetic heterogeneity. Diabetes 31: 1115-1118

6. Wagener DK, Sacks JM, LaPorte RE, MacGregor JM (1982) The Pittsburgh Study of insulin-dependent diabetes. Risk for diabetes among relatives of IDDM. Diabetes 31: 136-144

7. Li H, Isomaa B, Taskinen MR, Groop L, Tuomi T (2000) Consequences of a family history of type 1 and type 2 diabetes on the phenotype of patients with type 2 diabetes. Diabetes Care 23: 589-594

8. WHO Expert Committee on Diabetes Mellitus (1980) Second report. WHO Technical Report Series. 646. World Health Organization, Geneva

9. World Health Organization (1985) Diabetes Mellitus: Report of a WHO Study Group. WHO Technical Report Series No 727, World Health Organization, Geneva

10. The Expert Committee on the Diagnosis and Classification of Diabetes Mellitus (1997) Report of the expert committee on the diagnosis and classification of diabetes mellitus. Diabetes Care 20: 1183-1197

11. Alberti KGMM, Zimmet PZ (1998) Definition, diagnosis and classification of diabetes mellitus and its complications. Part 1: diagnosis and classification of diabetes mellitus. Provisional report of a WHO Consultation. Diabet Med 15: 539-553

12. Wilson RM, Van der Minne P, Deverill I et al. (1985) Insulin dependence: problems with the classification of 100 consecutive patients. Diabet Med 2: 167-172

13. Bingley PJ, Gale EAM (1989) The incidence of insulin-dependent diabetes in England: a study in the Oxford region 1985-6. BMJ 298: 558-560

14. Bingley PJ, Bonifacio E, Williams AJK, Genovese S, Bottazzo GF, Gale EAM (1997) Prediction of IDDM in the general population. Strategies based on combinations of autoantibody markers. Diabetes 46: 1701-1710

15. Verge CF, Stenger D, Bonifacio E et al. (1998) Combined use of autoantibodies (IA-2 autoantibody, GAD autoanti- body, insulin autoantibody, cytoplasmic islet cell antibodies) in type 1 diabetes: combinatorial islet autoantibody workshop. Diabetes 47: 1857-1866

16. Gillespie KM, Valovin SJ, Saunby J et al. (2000) HLA class II typing of whole genome amplified mouth swab DNA. Tissue Antigens 56: 530-538

17. Miller SA, Dykes D, Polesky H (1998) A simple salting out procedure for extracting DNA from human nucleated cells. Nucleic Acids Res. 16: 1215-1218

18. Bunce M, O'Neill CM, Barnado MCNM, Krausa P, Morris PJ, Welsh KI (1995) Phototyping: comprehensive DNA typing for HLA-A, B, C, DRB1, DRB3, DRB4, DRB5 and DQB1 by PCR with 144 primer mixes utilising sequence-specific primers (PCR-SSP). Tissue Antigens 46: 355-367

19. Verge CF, Howard NJ, Rowley MJ et al. (1994) Anti-glutamate decarboxylase and other antibodies at the onset of childhood IDDM: a population-based study. Diabetologia 37: $1113-1120$

20. Vandewalle CL, Falorni A, Lernmark A, Pipeleers DG, Gorus FK, Belgium Diabetes Registry (1995) High diagnostic sensitivity of glutamate decarboxylase autoantibodies in insulin-dependent diabetes mellitus with clinical onset between age 20 and 40 years. The Belgian Diabetes Registry. J Clin Endocrinol Metab 80: 846-851

21. Gorus FK, Goubert P, Semakula C et al. (1997) IA-2-autoantibodies complement GAD65-autoantibodies in newonset IDDM patients and help predict impending diabetes in their siblings. The Belgian Diabetes Registry. Diabetologia 40: 95-99

22. Savola K, Kulmala P, Vahasalo P, Ilonen J, Knip M (1998) Autoantibodies associated with type 1 diabetes mellitus persist after diagnosis in children. Diabetologia 41: 1293-1297

23. Decochez K, Weets I, Coolens JL et al. (2000) High frequency of persisting or increasing islet-specific autoantibody levels after diagnosis of type 1 diabetes presenting before 40 years of age. The Belgian Diabetes Registry. Diabetes Care 23: 838-844

24. Dromey JA, Mijovic CH, Christie MR, Patel A, Gill G, Barnett AH, Bain S (2000) HLA linked persistence of antibodies to GAD 65 in patients with 50 years of type 1 diabetes. Diabet Med 17: (Abstract)

25. Turner R, Stratton I, Horton V et al. (1997) UKPDS 25: autoantibodies to islet-cell cytoplasm and glutamic acid decarboxylase for prediction of insulin requirement in type 2 diabetes. Lancet 350: 1288-1293

26. Irvine WJ, McCallum CJ, Gray RS et al. (1977) Pancreatic Islet-cell antibodies in diabetes mellitus correlated with the duration and type of diabetes, coexistent autoimmune disease, and HLA type. Diabetes 26: 138-147

27. Irvine WJ (1977) Classification of idiopathic diabetes. Lancet i: $638-642$

28. Green A, Hougaard P (1983) Epidemiological studies of diabetes mellitus in Denmark: 4. Clinical characteristics of insulin-treated diabetes. Diabetologia 25: 231-234

29. Fagot-Campagna A, Narayan KMV, Imperatore G (2001) Type 2 diabetes in children. BMJ 322: 377-378

30. Gale EAM, Gillespie KM (2001) Diabetes and gender. Diabetologia 44: 3-15

31. Sachs JA, Cudworth AG, Jaraquemada D, Gorsuch AN, Festenstein H (1980) Type I diabetes and the HLA-D locus. Diabetologia 18: 41-43

32. Gatling W, Budd S, Walters D, Mullee MA, Goddard JR, Hill RD (1998) Evidence of an increasing prevalence of diagnosed diabetes mellitus in the Poole area from 1983-1996. Diabet Med 15: 1015-1021 
33. Amos AF, McCarty DJ, Zimmet P (1997) The rising global burden of diabetes and its complications: estimates and projections to the year 2010. Diabet Med 14: S1-S85

34. Morris AD, Boyle DIR, MacAlpine R et al. (1997) The diabetes audit and research in Tayside Scotland (DARTS) study: electronic linkage to create a diabetes register. BMJ 315: 524-528

35. Yudkin JS, Forrest RD, Jackson CA, Burnett SD, Gould MM (1993) The prevalence of diabetes and impaired glucose tolerance in a British population. Diabetes Care 16: 1530-1530
36. Macdonald MJ (1974) Equal incidence of adult-onset diabetes among ancestors of juvenile diabetics and non diabetics. Diabetologia 10: 767-773

37. Gale EAM (2001) The discovery of type 1 diabetes. Diabetes 50: 217-226

38. Molbak AG, Christau B, Marner B, Borch-Johnsen K, Nerup J (1994) Incidence of diabetes mellitus in age groups over 30 years in Denmark. Diabet Med 11: 650-655

39. Fajans SS, Cloutier MC, Crowther RL (1978) The Bating Memorial Lecture 1978. Clinical and etiologic heterogeneity of idiopathic diabetes mellitus. Diabetes 27: 1112-1125 